\title{
Experimental Animal Models of Fibrosis: Relevance and Considerations for Translational Health Research
}

\section{Bindu $S^{*}$}

Department of Zoology, Cooch Behar Panchanan Barma University, India

*Corresponding author: Samik Bindu, Department of Zoology, Cooch Behar Panchanan Barma University, Vivekananda Street, Cooch Behar, West Bengal 736101, India, Email: samikdot@gmail.com

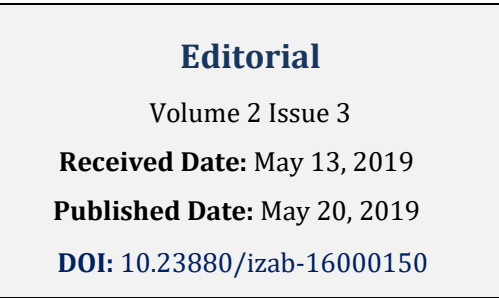

\section{Editorial}

Tissue fibrosis is a set of progressive debilitating diseases which although initiate as a normal wound healing response to an injury, end up with hyperactivated immunological state leading to overwhelming deposition of extra cellular matrix (ECM) components by activated fibroblasts. About $45 \%$ deaths in the US are attributed to fibrosis-associated organ damage [1]. Repeated cycles of tissue injury-repair cause molecular dyshomeostasis and chronic inflammation wherein the affected organs suffer stiffening, scarring and consequential functional loss. Fibrosis of major organs including lungs, heart, liver, kidney, intestine and skin largely contributes to the clinical complications of major diseases characterized by multi-organ failure. Various genetic factors are evidently linked to tissue-specific development of fibrosis in humans [2]. Owing to the severity of the disease and underlying molecular complexities, fibrosis has been one of the main stays of the pathological research. In order to understand the aberrantly activated pro-fibrotic molecular pathways along with identification of novel anti-fibrotic drug targets and therapeutic strategies, extensive in vitro and in vivo research has been under process wherein diverse experimental in vivo models of tissue fibrosis have been developed. Because fibrosis is mainly diagnosed in the later stages where the disease has already progressed and crossed the level of reversibility, experimental animal models remain the only option to kinetically follow the progression, besides identifying stage-specific biomarkers that can be prophylactically exploited for early diagnosis. These models are expected to mimic the pathogenesis in humans; however their relevance in terms of how precisely they simulate the human clinical conditions and predict the efficacy of experimentally established therapeutic strategies are always a matter of serious concern owing to the biochemical, metabolic and genetic differences between animals and humans. In addition, regulatory and ethical issues about inclusion of animals for translational health research further mandates rational usage following optimized standard operating procedures while considering the potential limitations. Talking about fibrosis, in spite of tissue-specific differences and diversity of inducers, epithelial hyperplasia and injury, fibroblast activation, metabolic reprogramming, persistent inflammation, aberrant epithelial-mesenchymal crosstalk and consequent excess ECM-components deposition are the molecular hallmarks that serve as the end point readouts while damaged tissue architecture and mortality serve as the predominant diagnostic indices $[3,4]$. Although spontaneous development and progression of fibrosis have been appreciated to different degrees in cats, dogs, chickens and horses, rodent models have always been most tractable and convenient owing to the 95\% similarity in genomic makeup of mice and humans and convenience in mice genome editing. BALB/c, C57BL/6 and Swiss albino are the most commonly used mice strains while Sprague Dawley, Zucker and Wistar are the most commonly used rat strains. It is worth considering that strain differences significantly influence the susceptibility to fibrosis in an organ-specific manner. Various animal models are used for studying the pathogenesis of fibrosis [5], although with widely evident inherent strain-specific responses [2]; for example while BALB/c mice show resistance to 


\section{International Journal of Zoology and Animal Biology}

pulmonary fibrosis (PF), they are instrumental in studying liver fibrosis. At the same time C57BL/6J mice are susceptible to PF and intestinal fibrosis while shows resistance to cardiac, hepatic and renal fibrosis. These differential outcomes owing to the genetic differences among the various in-bred strains underscore the influence of genetic makeup in disease outcome wherein epigenetic regulations via environmental factors add another level of complexity to the pathogenesis. Common animal models of experimental fibrosis have been discussed with critical comments regarding their relevance in terms of translational health research on tissue fibrosis.

PF is a grave clinical complication comprising $15 \%$ of the pulmonary physician's prescriptions. Owing to the multifactorial complex pathogenesis with limited therapeutic options, extensive studies on animal models are conducted to gain a clear insight of the molecular events leading to $\mathrm{PF}$ development and progression. Asbestosis, silicosis, bleomycin exposure, age-induced fibrosis, acid-instillation, radiation and transgenic models of TGF- $\beta$ and pro-inflammatory cytokine (IL13, TNF- $\alpha$, IL1 $\beta$ ) over expression are some of the experimental models of idiopathic pulmonary fibrosis (IPF) which largely contribute to the interstitial lung diseases characterized with usual pneumonia [5]. Among various agents, bleomycin (BLM) is the most commonly used fibrotic-inducer that largely simulates the pathogenic mechanisms in human and holds relevance due to its application as a potent anticancer drug. Resistance of $\mathrm{BALB} / \mathrm{c}$ mice to BLM-PF may in part be due to elevated BLM hydrolase in these species that metabolizes bleomycin to less toxic deamidoBLM (dBLM) form as well as reduced PARP activation and DNA damage upon BLM exposure [6]. Humanized mouse models are also under active exploration where intravenous instillation of human IPF-derive activated fibroblasts into non-obese diabetic/severe combined immunodeficiency (NOD/SCID/beige) mice are done. However, the progression of fibrosis in these immune deficient mice differs to a greater extent from the humans where inflammatory cells offer profound regulatory action on disease progression, thereby warranting a careful consideration before translating the results for predicting human outcomes. Mouse Genome Database has already listed 24 fibrosis-associated QTLs in mice of which 21 phenotypes are documented in lungs [2].

Scleroderma is another clinical complication characterized by multi organ fibrosis due to autoimmune response. Vascular injury and immune dysfunction are the early hallmarks. Experimental chicken models of vasculopathy have been widely explored which largely simulate the Raynaud's phenomena (observed in patients of systemic sclerosis) including microvascular occlusion and consequent erythema, edema and necrosis of the comb. Apart from chicken, the common murine model of skin fibrosis is the tight skin (Tsk) mouse model consisting of spontaneous in frame duplication of exons in the fibrillin-1 gene. Although skin manifestations are relevant with the clinical observations, pulmonary tissues of Tsk+/-mice show emphysematous phenotype instead of fibrotic phenotype. Other transgenic mice models are also largely used for studying skin fibrosis [7]. Notably, bleomycin mouse model is again relevant in skin fibrosis where repeated subcutaneous injections for at least 3 weeks are required compared to a single intra-tracheal instillation to induce pulmonary fibrosis. $\mathrm{C} 3 \mathrm{H} / \mathrm{He}$ and $\mathrm{B} 10 \mathrm{~A}$ are the best mice models in this case.

Moving to hepatic fibrosis (HF), cirrhosis and chronic hepatitis results in fibrotic damage and associated mortality. Viral infections, metabolic, toxic or obstructive damage causes fibrosis regardless of route of injury. Carbon tetrachloride-induced HF in BALB/c mice is the most common murine model, although other models of diet/chemical-induced hepatic injury are also explored where acetaminophen, thioacetamide, dimethyl and diethyl nitrosamine-enriched diet, methionine-choline deficient diet, ethionine-supplemented diet or even high fat diet have been tested in other mice models including A/J, BALB/cJ, AKR/J, C3H/HeJ, C57BL/6J, DBA/2J, $129 \times 1 / S v J W T, C 3 H / H e N, C 57 B L / 6 \mathrm{~N}$ and FVB/NJ to name a few with context-specific outcomes [8]. However for hepatitis C-induced liver damage, currently there is no murine model owing to the resistance of mice to human $\mathrm{HCV} / \mathrm{HBV}$. This led to the utilization of chimpanzees as model animals for studying HBV/HCV-induced hepatic fibrosis; although constraints like generation time, research budgets and larger facilities discouraged their use. Heterotopic human liver grafts and xenografting of human hepatocytes in immunodeficient rodents have also been followed as logical alternatives, although with specific limitations [9]. For alcohol-induced liver fibrosis baboons have been the best model organisms due to greater acceptance compared to rodents (with C57BL/6 mice being exception) with instinctive aversion to alcohol [10]. Non alcoholic fatty liver disease and steatohepatitis have been largely studied in C57BL/6 mice where high fat diet is instrumental besides methionine-deficient diet. Experiments on Obese (ob/ob) and Diabetic (db/db) mice have also yielded sufficient information on fatty liver development with varying degrees of endocrine and immune correlations. Finally for biliary fibrosis rats are better experimental models for surgical ligation of 


\section{International Journal of Zoology and Animal Biology}

common bile duct compared to mice due to their lack of gall bladder. The results of experimental hepatic fibrosis in animals considerably differ from the humans; yet they are beneficial for better understanding of the pathogenesis.

Chronic kidney disease (CKD) and diabetic nephropathy (DN) significantly account to end stage renal disease characterized by interstitial fibrosis, tubular atrophy and glomerulosclerosis. Murine models of renal fibrosis include unilateral ureteral obstruction (that mimics DN), intra-peritoneal administration of BSA, streptozotocin-induced diabetes and clamping the renal arteries to mention a few [11]. C57BL/6 mice mostly show resistance while 129S1/svImJ and CD1 mice are susceptible. Even the BALB/c mice are less sensitive to ureter clamping-induced fibrosis. In addition $\mathrm{db} / \mathrm{db}$ mice and Zuker rats are especially instrumental in studying diabetic nephropathy.

Intestinal fibrosis is yet another major clinical complication associated with Intestinal Bowel Disease (IBD) including ulcerative colitis and Crohn's disease. Most of the IBD animal models predominantly focused on the study of gut microbiota and immune responses. However recently focus has been shifted towards intestinal fibrosis per se which may be classified into various categories depending on the nature of inducers including gene-targeted, spontaneous, immunological, bacterial, chemical, radiation-associated and postoperative fibrosis [12]. Environmental factors have been especially instrumental in determining the outcomes. Intestinal epithelial cell injury, breach in the epithelial barrier and consequent activation of the mucosal immune system in response to microbial components initiate local inflammation that perpetuates into fibrosis. 2,4,6-trinitrobenzene sulfonic acid (TNBS), dextran sulfate sodium (DSS) and oxazolone are the most common chemical inducers of acute and chronic colitis and they largely reflect the nature of human IBD [13]. TNBS (together with ethanol) and oxazololne are administered by intra-rectal instillation while DSS is mixed in the drinking water. BALB/c mice are more susceptible to TNBS and oxazolone colitis while C57BL/6 is sensitive to DSS-induced chronic colitis. Other than mice rats are also susceptible to chemicals as well as intramurally injected peptidoglycanpolysaccharide (PG-PS) from bacterial cell wall. Direct administration in the bowel wall triggers transmural enterocolitis and subsequently chronic granulomatous inflammation which develops into fibrosis. Rats are also instrumental in understanding the development of fibrosis upon colonic wall-injection of fecal matter suspension and comparative analysis with TNBS colitis characterized by TGF- $\beta 1$ and collagen over production and intestinal stricture development [12]. These experimental models largely mimic the human pathological outcomes although the generic and interspecific differences in the immune responses should be strictly considered.

Finally cardiac fibrosis is yet one of the most severe clinical complication largely contributing to cardiac failure and mortality. Cardiac injury due to diverse causes including myocardial infarction, hypertrophy, pressure overload, repetitive ischemia, dilated cardiomyopathy and diabetic/obesity-induced cardiomyopathy results in cardiac fibrosis [14]. Diversely originating activated cardiac fibroblasts severely causes tissue scarring. Physical insults including cryogenic damage and coronary artery ligation and chemical damage by doxorubicin are some of the widely used experimental cardiac fibrosis models in mice and rats [15]. While 129S6, C57BL/6, FVB and Swiss albino mice are susceptible to coronary artery ligation-induced fibrosis, BALB/c mice are significantly fibrosis-resistant [16]. Again catecholamine and Isoproterenol-induced left ventricular hypertrophy and associated fibrosis was more profound in $\mathrm{A} / \mathrm{J}$ mice compared to C57BL/6J strain. Other than mice Sprague Dawley rats are very sensitive to cardiac fibrosis induced by either left coronary arterial ligation, chronic angiotensin II or aldosterone administration [15]. The results in murine models often mimic the human outcomes to a greater extent although the disparity between experimental fibrotic inducers and human physiological factors as well as the course of disease progression are to be considered.

Thus it is clear that experimental fibrosis in animals have greatly helped to understand the pathogenesis of the disease in human where early stages are mostly undetermined due to absence of acute symptoms. However it should be noted that owing to the molecular complexity, the immune responses and outcomes are greatly regulated by the nature of inducer and duration of exposure. Moreover environmental and genetic factors further add up to the tissue specific responses. Therefore experimental results observed in animals may be used for predicting human outcomes but with utter care considering the underlying species-specific responses.

\section{References}

1. Nanchahal J, Hinz B (2016) Strategies to overcome the hurdles to treat fibrosis, a major unmet clinical need. Proc Natl Acad Sci 113(27): 7291-7293. 


\section{International Journal of Zoology and Animal Biology}

2. Walkin L, Herrick SE, Summers A, Brenchley PE, Hoff CM, et al. (2013) The role of mouse strain differences in the susceptibility to fibrosis: a systematic review. Fibrogenesis Tissue Repair 6(1): 18.

3. Ho YY, Lagares D, Tager AM, Kapoor M (2014) Fibrosis--a lethal component of systemic sclerosis. Nat Rev Rheumatol 10(7): 390-402.

4. Hewlett JC, Kropski JA, Blackwell TS (2018) Idiopathic pulmonary fibrosis: Epithelialmesenchymal interactions and emerging therapeutic targets. Matrix Biol 71-72: 112-127.

5. Gharaee Kermani M, Ullenbruch M, Phan SH (2005) Animal models of pulmonary fibrosis. Methods Mol Med 117: 251-259.

6. Moeller A, Ask K, Warburton D, Gauldie J, Kolb M (2008) The bleomycin animal model: a useful tool to investigate treatment options for idiopathic pulmonary fibrosis?. Int J Biochem Cell Biol 40(3): 362-382.

7. Artlett CM (2014) Animal models of systemic sclerosis: their utility and limitations. Open Access Rheumatol 6: 65-81.

8. Yanguas SC, Cogliati B, Willebrords J, Maes M, Colle I, et al. (2016) Experimental models of liver fibrosis. Arch Toxicol 90(5): 1025-1048.

9. Delire B, Starkel P, Leclercq I (2015) Animal Models for Fibrotic Liver Diseases: What We Have, What We Need, and What Is under Development. J Clin Transl Hepatol 3(1): 53-66.
10. Lieber CS, Decarli LM (1976) Animal models of ethanol dependence and liver injury in rats and baboons. Fed Proc 35(5): 1232-1236.

11. Zeisberg M, Soubasakos MA, Kalluri R (2005) Animal models of renal fibrosis. Methods Mol Med 117: 261272.

12. Rieder F, Kessler S, Sans M, Fiocchi C (2012) Animal models of intestinal fibrosis: new tools for the understanding of pathogenesis and therapy of human disease. Am J Physiol Gastrointest Liver Physiol 303(7): G786-801.

13. Wirtz S, Popp V, Kindermann M, Gerlach K, Weigmann B, et al. (2017) Chemically induced mouse models of acute and chronic intestinal inflammation. Nat Protoc 12(7): 1295-1309.

14. Rai V, Sharma P, Agrawal S, Agrawal DK (2017) Relevance of mouse models of cardiac fibrosis and hypertrophy in cardiac research. Mol Cell Biochem 424(1-2): 123-145.

15. Sun Y, Weber KT (2005) Animal models of cardiac fibrosis. Methods Mol Med 117: 273-290.

16. Van den Borne SW, van de Schans VA, Strzelecka AE, Vervoort-Peters HT, Lijnen PM, et al. (2009) Mouse strain determines the outcome of wound healing after myocardial infarction. Cardiovasc Res 84 (2): 273-282. 